\title{
Well-Being in Times of Crisis: Interdisciplinary Evidence and Policy Implications
}

\author{
Antonella Delle Fave
}

(C) Springer Science+Business Media Dordrecht 2014

The papers presented in this special section of the Journal of Happiness Studies share a common goal: the assessment of subjective well-being (SWB) in times of crisis. This goal was pursued through the interdisciplinary lens that characterizes the Journal. Two of the studies focus on the national changes in SWB following the recent global economic crisis; another one deals with the challenges posed to policy makers by the ongoing demographic transition; the remaining three papers explore the issue at the psychological level, investigating well-being fluctuations in the face of disruptive events or circumstances.

In the first paper, Helliwell, Huang and Wang discuss the role of social capital in buffering the negative consequences of economic crises on SWB, taking the 2007-2008 international crisis as an exemplary case. They draw evidence from a variety of sources: the Gallup-Healthways survey, that since 2008 provides daily assessments of well-being among US citizens; multiple waves of data collected in South Korea and in several other OECD countries, divided into three groups according to the negative, flat or positive trajectory of happiness levels showed during the years 2008-2011; five rounds of data (2002-2010) derived from the European Social Survey and collected in transition and nontransition countries. Across the databases, the relationship between social capital and SWB clearly emerged. More specifically, in US metropolitan areas characterized by higher social engagement the levels of happiness were also higher. In South Korea, the specific policy strategies undertaken by the government to counterbalance the economic crisis, based on solidarity and social sharing, were associated with increases in the happiness levels that could not be predicted by greater income only. In those OECD countries that were severely hit by the economic crisis, the decline in happiness levels-greater than expected on the basis of the GDP drop-was partially related to a substantial decrease in social and institutional trust. Finally, the political transition occurring in Eastern European countries during the last decade fostered higher levels of social trust, that directly contributed to a global increase of happiness levels, in spite of the negative effects of economic drawbacks.

\footnotetext{
A. Delle Fave $(\bowtie)$

Department of Pathophysiology and Transplantation, University of Milano, via F. Sforza, 35, 20122

Milan, Italy

e-mail: antonella.dellefave@unimi.it
} 
The issue of the recent economic crisis is also addressed by Christian Bjørnskov, who explores the role and impact of government interventions in buffering the negative consequences of economic crises on satisfaction with life levels. In the paper two major economic perspectives are juxtaposed: the Keynesian view, that claims the need for centralized institutional reforms to correct market failures; and the Austrian and public choice schools' approach, assuming that governments will never get sufficient information to design adequate reforms, and thus they should provide markets with adequate levels of flexibility and freedom to pursue an effective balance in the face of economic changes. In this study, life satisfaction levels are analyzed across European countries from 1975 to 2011, based on the EuroBarometer survey data. In line with the study by Helliwell and colleagues, findings confirm that life satisfaction is higher in countries where social trust levels are higher as well. Moreover, results suggest that crisis-related drops in life satisfaction are lower in countries with lighter regulatory burdens, compared with countries where market regulations are heavier.

The recent economic crisis has also generated a lively debate around the sustainability of welfare systems across countries. This problem is especially evident as concerns provisions for the elderly. In most countries the proportion of retired people is rapidly growing, and this trend imposes a mounting financial pressure that will become unsustainable in the next few decades. One of the strategies available to governments in order to face this problem is to postpone retirement at an older age. Elizabeth Mokyr Horner addresses this issue by investigating the relationship between age of retirement and SWB. Findings were obtained from a large cross-sectional database comprising information about men aged 50-70, living in European countries and the United States. The study shows that the age of retirement does not substantially impact on SWB levels in the long run. More specifically, both early and normal retirement periods are associated with a significant increase in SWB, however followed by a decline after few years. These findings have important implications for policy makers, suggesting that a later retirement would not entail negative consequences for the SWB fluctuation pattern typically characterizing the last part of the life.

A different kind of crisis is addressed by Uchida, Takahashi and Kawahara. From a psychological perspective, they analyze the impact on perceived well-being of the earthquake that affected the Eastern part of Japan in March 2011, whose devastating consequences included a tsunami as well as the breakdown of the Fukushima nuclear plant. Longitudinal information on hedonic and eudaimonic dimensions of well-being was collected before and after the disaster among young adults living in non-affected areas. Findings highlighted an apparent paradox: well-being levels increased after the disaster, especially among youth who reported being concerned with the event and its consequences. More specifically, this group of participants showed an increase in SWB after the earthquake, despite a parallel increase in temporary negative emotions. They also reported a positive change in attitude towards ordinary life and connectedness, as well as higher engagement in prosocial behavior compared with the participants who reported low concern with the impact of the event on their country and fellow citizens.

The last two articles are interconnected. They aim at providing empirical evidence supporting the homeostatic model of SWB, in view of its potential for application to counterbalance the negative consequences of challenging conditions. Cummins, Li, Wooden and Stokes propose to interpret the substantial individual stability of SWB values-repeatedly detected in longitudinal studies-according to the homeostatic perspective. The basic theoretical assumption underlying their work is that the observed stability of SWB levels should be ascribed to individual differences in the SWB set-point, a 
genetically determined measure that represents the homeostatic level of well-being, typical of normal life conditions. Longitudinal assessments of mood and life satisfaction derived from the Australian HILDA survey 2001-2010 are analyzed with the aim to identify individual SWB set-points, from which well-being levels may deviate according to life challenges or disruptive events. It is also hypothesized that most challenge-related deviations are temporary, and fall within a limited range of values under homeostatic control. This guarantees the possibility to restore normal SWB levels once the problem is solved or the challenge removed. Findings show that the majority of participants' SWB set-points are comprised between 75.5 and 80 on a 1-100 scale, following a normal distribution pattern consistent with the hypothesis of a genetically determined feature. In the following paper, Cummins and Wooden discuss the potential of this perspective in explaining the changes in SWB levels occurring in the face of life challenges varying in severity. More specifically, low-impact and transient challenges are expected to produce deviations from the set-point comprised within the homeostatically controlled range, thus allowing for resilience processes and set-point restoration. On the contrary, highly disruptive events may lead to greater deviations in SWB values, not controllable anymore through homeostatic mechanisms. In these cases, external resources such as social and financial support should be provided in order to restore homeostatic levels of SWB.

Overall, the papers in this special section share a crucial finding. Across the variety of disruptive events and circumstances taken into account-economic crises, retirement, natural disasters - results highlight the importance of social relations, social trust and connectedness in buffering the negative consequences of life and social challenges on SWB.

This finding is consistent with theories and models emphasizing the centrality of social connectedness for well-being promotion (Deci and Ryan 2000; Forgeard et al. 2011; Keyes 1998; Marujo and Neto 2014; Pöhlman 2001; Ryff and Singer 2008). The innate need to belong to social groups and to find meaning in the relationship with others, as well as the crucial role of social connections for human survival and reproduction lead individuals across cultures to invest a large amount of psychological and material resources in building and cultivating relations (Baumeister and Leary 1995; Delle Fave et al. 2013; Jablonka and Lamb 2005; Stillman et al. 2009). Theories and empirical studies on resilience have highlighted the protective role of family and social connections in promoting successful adjustment under difficult circumstances (Walsh 2003; Luthar 2006; Simon et al. 2008; Scrignaro et al. 2009; Zautra et al. 2012). Emergency situations such as natural disasters repeatedly proved to be effective in mobilizing collective agency and responsibility (Quarantelli 1985). The relevance of relationships for well-being is also emphasized by studies on altruism and prosocial behavior, that have recently highlighted the positive consequences of helping others for both the beneficiary and the benefactor (Musick and Wilson 2003; Levine et al. 2008; Schwartz et al. 2003; Soosai-Nathan et al. 2013; Weinstein and Ryan 2010).

However, besides providing further support to previous evidence, the findings illustrated in this special section substantially contribute to indicating a pathway-one of the few ones; maybe the only one?- to cope with the increasingly limited resources of nations and communities worldwide. The sustainability of individual and collective well-being is grounded into solidarity and sharing. The availability of a strong and solid relationship network may prevent individual SWB levels from deviating beyond the homeostatic range under highly challenging circumstances, and the network implementation can help restore SWB point after failure produced by disruptive events. 
Nevertheless, the strategies to achieve these goals may change with time and across communities. The South Korean approach to unemployment illustrated by Helliwell and colleagues may not be straightforwardly applicable in more individualistic contexts; similarly, the diversity of retirement provisions across countries described by Mokyr Horner may generate different solutions to the increasing fiscal pressures related to the global population aging trend. This consideration implies the admission of a major limitation in well-being studies, that must be still overcome through systematic theoretical and empirical efforts across disciplines: the role of culture in the promotion of well-being and in the development of related social policies should be more thoroughly investigated. This topic includes several thorny issues, such as the need for distinguishing between nations and cultures, often misleadingly equated. Despite the globalization trend, cultural differences are still alive, and they substantially contribute to that precious diversity of worldviews and solutions to human problems that provided our species with flexibility and creative adaptation along the millennia. More resources should be invested by social scientists and institutions in the effort to understand cultural convergences and differences, with the aim of fruitfully capitalizing on diversity to build a more complex and integrated global community.

\section{References}

Baumeister, R. F., \& Leary, M. R. (1995). The need to belong: Desire for interpersonal attachments as a fundamental human motivation. Psychology Bulletin, 117, 497-529.

Deci, E. L., \& Ryan, R. M. (2000). The 'what' and 'why' of goal pursuits: Human needs and the selfdetermination of behavior. Psychological Inquiry, 11, 227-268.

Delle Fave, A., Brdar, I., Wissing, M. P., \& Vella-Brodrick, D. (2013). Sources and motives for personal meaning in adulthood. The Journal of Positive Psychology, 8, 517-529.

Forgeard, M. J. C., Jayawickreme, E., Kern, M. L., \& Seligman, M. E. P. (2011). Doing the right thing: Measuring well-being for public policy. International Journal of Well-being, 1, 79-106.

Jablonka, E., \& Lamb, M. J. (2005). Evolution in four dimensions. Genetic, epigenetic, behavioural and symbolic variations in the history of life. Cambridge, MA: MIT Press.

Keyes, C. L. M. (1998). Social well-being. Social Psychology Quarterly, 61, 121-140.

Levine, R. L., Reysen, S., \& Ganz, E. (2008). The kindness of strangers re-visited: A comparison of 24 US cities. Social Indicator Research, 85(3), 461-481.

Luthar, S. S. (2006). Resilience in development: A synthesis of research across five decades. In D. Cicchetti \& D. J. Cohen (Eds.), Developmental psychopathology: Risk, disorder, and adaptation (2nd ed., Vol. 3, pp. 739-795). Hoboken: Wiley.

Marujo, H., \& Neto, L. M. (Eds.). (2014). Positive nations and communities. Dordrecht: Springer.

Musick, M. A., \& Wilson, J. (2003). Volunteering and depression: The role of psychological and social resources in different age groups. Social Sciences \& Medicine, 56(2), 259-269.

Pöhlman, K. (2001). Agency and communion-orientation in life goals: Impacts on goal pursuit strategies and psychological well-being. In P. Schmuck \& K. Sheldon (Eds.), Life goals and well-being: Towards a positive psychology of human striving (pp. 68-84). Ashland, $\mathrm{OH}$ : Hogrefe \& Huber.

Quarantelli, E. L. (1985). An assessment of conflicting views on mental health. The consequences of traumatic events. In C. R. Figley (Ed.), Trauma and its wake (pp. 173-215). New York: Brunner/ Mazel.

Ryff, C. D., \& Singer, B. H. (2008). Know thyself and become what you are: A eudaimonic approach to psychological well-being. Journal of Happiness Studies, 9, 13-39.

Schwartz, C. E., Meisenhelder, J. B., Ma, Y., \& Reed, G. (2003). Altruistic social interest behaviors are associated with better mental health. Psychosomatic Medicine, 65, 778-785.

Scrignaro, M., Barni, S., \& Magrin, E. (2009). The combined contribution of social support and coping strategies in predicting post-traumatic growth: a longitudinal study on cancer patients. PsychoOncology, 20, 823-831.

Simon, J., Murphy, J., \& Smith, S. (2008). Building resilience: Appreciate the little things in life. British Journal of Social Work, 38, 218-235. 
Soosai-Nathan, L., Negri, L., \& Delle Fave, A. (2013). Beyond pro-social behaviour: An exploration of altruism in two cultures. Psychological Studies. 10.1007/s12646-013-0184-Z.

Stillman, T. F., Baumeister, R. F., Lambert, N. M., Crescioni, A. W., DeWall, C. N., \& Fincham, F. D. (2009). Alone and without meaning: Life loses meaning following social exclusion. Journal of Experimental and Social Psychology, 45, 686-694.

Walsh, F. (2003). Family resilience: A framework for clinical practice. Family Process, 42, 1-18.

Weinstein, N., \& Ryan, R. M. (2010). When helping helps: Autonomous motivation for prosocial behaviour and its influence on well-being for the helper and recipient. Journal of Personality and Social Psychology, 98(2), 222-244.

Zautra, A. J., Stuart Hall, J., \& Murray, K. E. (2012). Resilience. A new definition of health for people and communities. In J. W. Reich, A. J. Zautra, \& J. Stuart Hall (Eds.), The handbook of adult resilience (pp. 3-29). New York: The Guilford Press. 